Revue d'histoire de l'Amérique française

REVUE D.HISTOIRE DE L'AMÉRIQUE FRANÇAISE

KEATING, Peter, La science du mal. L'institution de la

psychiatrie au Québec, 1800-1914. Montréal, Boréal, 1993. 204 p. $22,95 \$$

\title{
André Cellard
}

Volume 48, numéro 1, été 1994

URI : https://id.erudit.org/iderudit/305306ar

DOI : https://doi.org/10.7202/305306ar

Aller au sommaire du numéro

Éditeur(s)

Institut d'histoire de l'Amérique française

\section{ISSN}

0035-2357 (imprimé)

1492-1383 (numérique)

Découvrir la revue

Citer ce compte rendu

Cellard, A. (1994). Compte rendu de [KEATING, Peter, La science du mal. L'institution de la psychiatrie au Québec, 1800-1914. Montréal, Boréal, 1993. 204 p. 22,95 \$]. Revue d'histoire de l'Amérique française, 48(1), 95-97.

https://doi.org/10.7202/305306ar d'utilisation que vous pouvez consulter en ligne.

https://apropos.erudit.org/fr/usagers/politique-dutilisation/ 
KEATING, Peter, La science du mal. L'institution de la psychiatrie au Québec, 1800-1914. Montréal, Boréal, 1993. 204 p. 22,95\$

Bien qu'il convienne mieux à un ouvrage de théologie, c'est sous le titre étrange de «La science du mal» que Peter Keating a fait paraître sa thèse de 
doctorat qui porte sur l'avènement de la psychiatrie au Québec. Ce titre est d'autant plus inattendu que l'ouvrage de Keating ne traite pas de la folie ou des fous au plan des perceptions négatives teintées d'immoralité qui s'y rattachent de plus en plus au XIX ${ }^{\mathrm{e}}$ siècle (le «mal»), mais aborde plutôt la question sous l'angle de l'étude de l'apprentissage d'un nouveau savoir médical (car il ne saurait être, bien sûr, question de «science» ici). En privilégiant une telle approche, Peter Keating a donc décidé de mettre de côté l'objet de la psychiatrie, soit les fous et le mal qui les afflige. Il a aussi choisi d'ignorer le champ des représentations de la folie, de même que toute la dimension du rôle des pouvoirs publics dans l'avènement de l'asile et de la gestion des troubles mentaux, pour se concentrer sur son traitement et ceux qui s'en disent désormais les experts. Cette démarche est bien entendu tout à fait légitime et même nécessaire, et l'angle sous lequel Keating aborde son sujet ne manque pas d'intérêt.

Dans son ouvrage, l'auteur a d'abord cherché à se distancer de la perspective dite critique qui analyse l'avènement de l'asile et du traitement qui l'accompagne, à travers le prisme de la mise en place des principales mesures de régulation sociale suscitées par la transition au capitalisme. Or, cette approche a fortement lié la naissance de la psychiatrie à celle de l'asile. Comme l'aventure asilaire s'est soldée par un échec lamentable, certains auteurs en ont aussi fait l'échec de la psychiatrie. Peter Keating s'élève ici contre cette approche, et procède en quelque sorte à une réhabilitation de la psychiatrie en dissociant le développement du traitement moral - thérapie précurseure de la psychiatrie - de celui de l'asile. C'est ainsi que, selon lui, l'avènement de ce traitement se serait fait indépendamment de l'asile, et l'auteur en a pour preuve l'apparition dudit traitement moral au Québec dans un contexte hospitalier plutôt qu'asilaire et ce, dès la première moitié du $\mathrm{XIX}^{\mathrm{e}}$ siècle (avant même la naissance de l'asile donc). Il s'agit d'une affirmation importante dans la reconstruction de l'auteur, et c'est pourquoi il intitule une section de son ouvrage «La mise en place du traitement moral dans les hôpitaux du Québec: 1800-1824». Malheureusement, l'auteur ne possède pas les assises empiriques nécessaires à une telle affirmation. Premièrement, les quelques allusions à la pratique, bien hypothétique, d'un quelconque traitement moral au Québec avant l'avènement de l'asile ne concernent exclusivement qu'une seule institution, soit l'Hôpital général de Québec. Or il y a, à cette époque, tout un monde qui sépare les hôpitaux généraux, hospices semi-carcéraux qui n'ont d'hôpital que le nom, des hôtels-Dieu, ancêtres de nos hôpitaux modernes. Cela dit, nous ne sommes pas opposé, a priori, à l'hypothèse d'une tentative d'expérimentation du traitement moral à l'Hôpital général de Québec au début des années 1820. Cependant, si tel fut bien le cas, il n'a pu s'agir que d'une tentative fort modeste, limitée dans le temps et sans conséquence, puisque l'on ne retrouve par la suite aucune trace ou allusion à une quelconque pratique du traitement moral au Bas-Canada avant l'avènement de l'asile. Mais par-dessus tout, force est de constater que l'auteur ne possède pas de preuves documentaires directes permettant d'affirmer que le traitement moral fut pratiqué à l'Hôpital général de Québec. Il y a bien un docteur Holmer qui affirme à une com- 
mission d'enquête que des instructions ont été données aux religieuses de pratiquer le traitement moral dans les cellules de cette institution, mais cette affirmation sera par la suite fortement démentie par de nombreux contemporains, soit: un autre médecin de l'institution, le docteur Hackett; un comité visiteur; le docteur Frémont (pionnier de l'aliénisme et du traitement moral au Québec) qui jugera cette affirmation ridicule, de même que les religieuses de l'Hôpital général elles-mêmes (Cellard, 1991: 172-179).

En ce qui a trait à la naissance de l'institution asilaire au Québec, nous croyons que Peter Keating fait fausse route lorsqu'il affirme que Colborne nomma en 1837 une commission chargée de la mise sur pied d'un asile temporaire. C'est avec l'assentiment de son Comité Spécial (qui ne fut formé qu'en 1838) que Colborne prit cette décision au printemps 1839, comme le laissent voir clairement le procès-verbal de la réunion et le certificat de nomination des neuf commissaires ${ }^{1}$.

La troisième partie de l'ouvrage de Keating traite des premières années des institutions asilaires québécoises. Il nous fait voir, entre autres, comment le premier asile permanent de Beauport fut affecté par le problème des incurables. La quatrième partie de l'ouvrage porte surtout sur la crise de l'asile et de l'expertise psychiatrique lors des années 1880 . Le livre se termine sur un intéressant chapitre qui concerne l'institutionnalisation et la professionnalisation de la psychiatrie qui devient spécialité médicale au tournant du siècle. L'auteur y fait aussi un survol de l'enracinement au Québec d'une doctrine psychiatrique - la dégénérescence. C'est vraiment à cette époque que certaines théories de la psychiatrie prennent de la profondeur. La psychiatrie s'institutionnalise alors de façon décisive avec le développement de l'enseignement à Québec et à Montréal et celui de «l'expertise» médico-légale.

Somme toute, Keating a produit un ouvrage intéressant qui retrace certains moments des premiers balbutiements de ce que l'on appellera plus tard la psychiatrie. On y suit, tout au long du XIX ${ }^{\mathrm{e}}$ siècle, les principaux cheminements fondateurs qui feront de cette «science» un nouveau savoir médical. Et malgré certains éléments sur lesquels nous avons exprimé notre désaccord, il n'en demeure pas moins qu'il s'agit ici sans conteste d'une contribution importante à l'histoire de la médecine au Québec.

Départements de criminologie et d'histoire Université d'Ottawa

ANDRÉ CELLARD

1. Voir A. Cellard et D. Nadon, «Ordre et désordre: le Montreal Lunatic Asylum et la naissance de l'asile au Québec», Revue d'histoire de l'Amérique française, 39,3 (hiver 1986): 353. Lorsqu'il affirme que cette commission a été mise sur pied en 1837, Keating s'appuie sur une source indirecte. Il s'agit d'un vague passage d'une pétition de citoyens de Montréal qui prétend quelques années plus tard que Colborne aurait nommé cette commission en 1837. Or, la mémoire de la personne qui a rédigé la pétition a tout simplement fait défaut. 